\title{
Entre recette magique d'Al-Bûnî et prière islamique d'al-Ghazali : textes talismaniques d'Afrique occidentale.
}

Between al-Bûnî's Magical Recipes and al-Ghazâlî's Islamic Prayer: Talismanic Texts from Western Africa"

\section{Constant Hamès}

\section{OpenEdition Journals}

Édition électronique

URL : http://journals.openedition.org/span/1344

DOI : $10.4000 /$ span. 1344

ISSN : 2268-1558

Éditeur

École pratique des hautes études. Sciences humaines

\section{Édition imprimée}

Date de publication : 1 novembre 1993

Pagination : 187-223

ISSN : 0294-7080

\section{Référence électronique}

Constant Hamès, «Entre recette magique d'Al-Bûnî et prière islamique d'al-Ghazali : textes

talismaniques d'Afrique occidentale. », Systèmes de pensée en Afrique noire [En ligne], 12 | 1993, mis en ligne le 03 décembre 2013, consulté le 10 décembre 2020. URL : http://journals.openedition.org/span/ 1344 ; DOI : https://doi.org/10.4000/span. 1344 


\section{ENTRE RECETTE MAGIQUE D'AL-BÛNÎ ET PRIÈRE ISLAMIQUE D'AL-GHAZÂLÎ: TEXTES TALISMANIQUES D'AFRIQUE OCCIDENTALE}

par

\section{Constant Hamès}

En travaillant sur un corpus d'une vingtaine de recettes magiques a base de textes islamiques dont se servait un moodi ("marabout") soninké (Hamès, 1987), la question de la provenance de ces recettes s'est posée. Le moodi lui-même ne les avait pas inventées et les gens de sa famille dont il les tenait, particulièrement son père, non plus. Alors qui? et d'où?

La tradition orale, la consultation de certains fonds de manuscrits et l'ouvrage déja ancien d'E. Doutté (1908) sur le Maghreb font émerger, de l'anonymat apparent de tous ces textes magiques d'Afrique occidentale, un nom, celui d'al-Bûnî et le nom d'un de ses ouvrages, le Shams al-macârif, "Le soleil des connaissances" .

Les comparaisons entre cet ouvrage et les recettes talismaniques de "terrain" s'avèrent tout à fait instructives. On y regardera donc de près. Cependant, cette comparaison n'est pas totalement satisfaisante car plusieurs textes talismaniques recueillis ne coïncident pas - ou trop

${ }^{1} \mathrm{Cf}$. à ce sujet les travaux récents de $\mathrm{P}$. Lory.

Fétiches II. Puissance des objets, charme des mots, systèmes de pensée en Afrique noire, 12, 1993 
partiellement - avec le modele général proposé par al-Bûnî. Par contre ils ressemblent à ces formes de prière que les traditions d'origine de l'islam ont dénommées $d u^{c} \hat{a}, \mathrm{pl} . d a^{c}$ wât, c'est-à-dire "appel", "invocation", "supplique" etc. Or une des présentations les plus systématiques de ces $d a^{c}$ wât est contenue dans l'œuvre maîtresse d'al-Ghazâlî, l'Ihyâ' 'ulâm ad-dîn (La revivification des sciences de la religion) (Al-Ghazâlî, s.d.). Là aussi, la confrontation avec les écrits africains pourra nous indiquer s'il s'agit d'un second modèle d'inspiration possible.

Pour fixer les idées, prenons deux exemples de terrain, provenant du corpus soninké et correspondant apparemment à des univers de référence différents.

Premier exemple. Il s'agit de deux courtes recettes pour agir sur les paroles d'un adversaire ou d'un supérieur en lui "prenant la bouche". Voici les textes.

"Recette de l'adversaire (al-khasam) ou d'un chef (sultân). Si tu veux prendre la bouche de celui qui est furieux contre toi (yaghdabu calayka) ou si tu veux arriver à tes fins auprès de celui que tu sollicites, recopie ce tableau (al-khâtim), glisse-le dans un étui (unbab) que tu maintiendras d'un fil ou d'une ficelle puis mets-le en poche: il aura pour effet de faire se mélanger les paroles dans la bouche de ton adversaire et de l'empêcher de trouver le bon argument, in shấ'. (Une écriture différente a hâtivement rajouté "Allah", ce qui, au lieu de "si veut" donne "si Allah le veut"). Voici le tableau à transcrire sans bismillah (bilâ basmalat)."

Celui-ci contient une formule verbale non identifiée, entrecoupée par un des signes "cabalistique" - IIII - qu'al-Bûnî appelle parfois tilasm, suivie d'une ligne et demie d'un verset coranique dont il manque le dernier terme verbal: "Sourds, muets, aveugles, ils.... ne pas" (Coran, II, 18 \& 171). La négation (lâ) est répétée sept fois. Le contexte du verset, dans le Coran, désigne ceux qui sont abandonnés par Allah. Mais nous savons d'expérience que ce n'est pas le contexte que vise le talisman mais bien le sens premier et littéral des termes qu'il a sélectionnés. 
Une deuxième recette suit:

De même, avec le tableau suivant, tu prends la bouche de l'adversaire (fam al-khasam) et de ce fait, tu le domines (taghlibuhu). Voici le tableau, à mettre en poche.

Le tableau est un carré de 3 x 3 cases (appelé généralement pour cette raison mutallat $)$; la case centrale est vide, fort probablement réservée à l'inscription du nom de l'adversaire; les trois cases supérieures portent chacune un terme dont l'ensemble donne: "Il fut trouble celui qui était infidele" (Cor, II, 258); les cinq autres cases comportent des chiffres.

Dans les deux cas, on est en présence d'une magie de type sympathique puisqu'il s'agit de transférer sur quelqu'un les effets du contenu explicite d'un texte efficace ("sourds, muets, aveugles" "trouble"). Il s'agit aussi de magie maléfique si l'on en juge par la charge agressive du projet et du désordre psycho-moteur souhaité. Fait remarquable, contrairement à l'accoutumée, aucune formule islamique pieuse n'introduit la recette et il est même spécifié qu'il faut l'en écarter: "bilâ basmalat"; le "in shâ"" (si veut) non suivi d'Allah dans l'écriture originale est peut-être symptomatique.

On peut alors s'interroger sur le statut du texte coranique tel qu'il est utilisé ici: quelle est la "force" efficace à laquelle le talisman se réfere? Une indication intéressante vient de ce qu'al-Bûnî a traité la même question de l'action magique sur la parole d'autrui, au moyen du même vocabulaire coranique. Sa recette (Shams, II, 223) vise en effet "à nouer les langues ( $l i{ }^{c}$ aqd al-alsina), entre autres en recopiant cinq fois les mots "sourds, muets" et sept fois "aveugles" suivis de "et ils ne voient pas, ne s'expriment pas, ne parlent pas " (Cor, VII, 179) puis, s'adressant à la personne visée: "ô un tel fils d'une telle (ben fulâna $)^{2}$, ta langue est nouée." Une allusion et un appel analogiques

${ }^{2}$ Alors que la société arabe est patrilinéaire, la magie arabo-musulmane, comme toute magie semble-t-il, s'intéresse à la filiation par les fermmes. Le prototype, dans notre corpus soninké, en est l'appel au nom de la mère de Moïse. Ayant fait assister un jour un ami marabout à une messe catholique à la campagne, il voulut discuter avec le curé à qui il demanda à brûle-pourpoint s'il connaissait le nom de la mère de Moïe... malgré ses encyclopédies, le curé ne le trouva pas alors que le marabout le connaissait fort bien à cause des talismans. 
sont faits à "Allah qui a noué les sept ( $s a b^{c} a$ ) cieux et les lions ( $\operatorname{sib} \hat{a}^{c}$ ) de Daniel" puis al-Bûnî propose l'utilisation d'une graphie originale des trois termes coraniques "sourds, muets, aveugles".

La parenté entre la première recette de terrain et celle d'al-Bûnî apparaît clairement (nouer la langue; sourds, muets, aveugles, ne....pas; le chiffre sept). Dans le même sens, on relèvera la construction du $2 \mathrm{e}$ tableau qui mêle mots et chiffres. Al-Bûnî est particulièrement connu pour la prescription et l'exploitation systématiques de ce type de tableau, comme l'atteste son ouvrage, le Shams, notamment dans sa quatrieme partie.

Deuxième exemple. Voici d'abord une prescription à base de récitations pieuses, pour le rachat de fautes morales.

"Talisman. Les Khalifes ont rapporté d'après le messager d'Allah - le salut d'Allah et la paix soient sur lui - qu'il existe cinq invocations pieuses (adkâr) à faire pour le rachat de ses fautes (fidya). La première: il n'y a de dieu qu'Allah, à réciter 70000 fois. La deuxième: loué soit Allah, louange à lui, loué soit Allah, le glorieux, à réciter 1000 fois. La troisième: au nom d'Allah le clément, le miséricordieux, à réciter 800 fois. La quatrième: dis, il est lui, Allah, l'unique, à réciter 50 fois. La cinquième: le salut sur le Prophète, soit la formule: Que le salut d'Allah et la paix soient sur lui, 12000 fois. Fin."

Comparativement aux "recettes" précédentes, la tonalité est différente. La présentation formelle suit le modèle des traditions prophétiques (hadît) et les formules à réciter s'inspirent de celles des confréries islamiques ( $\underline{d i k r}, \mathrm{pl}$. adkâr) et, au-delà, d'une tradition mystique dont al-Ghazâlî représente le pivot. On remarquera cependant l'importance des chiffres (il y a cinq invocations), des nombres et des répétitions. Voici encore, dans un registre voisin, une récitation coranique (Cor, III, 6) à faire dans une situation bien particulière:

"Talisman de l'enfant. A réciter au moment de l'accouplement (cind al-jama $\hat{a}^{c} a$ ). La femme trouvera un enfant. 'C'est lui qui vous fait prendre forme dans les utérus, selon sa volonté. Il n'y a pas de dieu en-dehors de lui, l'aimé, le sage'. Fin." 
Il n'y a pas de rituel, pas d'intrumentation à mettre en ceuvre sinon la récitation au moment opportun de ce verset coranique dont on veut s'accaparer le sens et les effets d'ordre sympathique ${ }^{3}$.

Magie d'un côté, prière de l'autre? Acceptons pour le moment de poser la question en ces termes puisque les différences entre des talismans effectivement utilisés en Afrique de l'ouest nous y invitent et examinons de plus près les modèles à la fois historiques et paradigmatiques de ces deux types de recours.

\section{La magie de type al-Bûnî}

Le cas le plus célèbre et le plus populaire mais en même temps le plus ambigu de ce type de magie est celui fourni par le même Abû Hamîd Muhammad al-Ghazâlî (1058-1111) qui rapporte, dans son ouvrage de facture autobiographique $\mathrm{Al}$-munqi $\underline{\text { d }}$ min ad-dalal ("Sortir de l'impasse") un exemple d'application d'un carré magique lors d'un accouchement difficile.

Le contexte est celui d'une argumentation ad hominem menée par al-Ghazâlî en faveur de la reconnaissance de la nature spécifique de la Prophétie, "œil qui perçoit des choses singulieres au-delà des limites de la raison (tawr warâ' al- ${ }^{c} a q l$ )". De la même façon, plaide-t-il, le rituel de la shart ${ }^{c} a$, celui du nombre de prosternations ( $\left.r a k a^{c} \hat{a} t\right)$ durant la priere par exemple, possède des "vertus" cachées, "qui ne sont pas accessibles à la logique rationnelle (mâ la yudrik bi-l-hikmat al- $\left.{ }^{c} a q l i ́ y a\right)$ et qui ne peuvent être perçues qu'avec l'ail de la Prophétie (illâ $b i-^{c}$ ayn an-nubawwa)". L'argument ad hominem va consister à s'adresser aux incrédules sur le terrain de leur propre spécialisation intellectuelle ou professionnelle et al-Ghazâlî cite nommément "l'astronomie,

${ }^{3}$ Ceci n'est exact que si on suit la recette à la lettre. Dans la pratique, on peut penser que la formule n'est pas toujours récitée oralement mais plutôt portée en amulette, disposée sous le lit, etc. La majorité des paysans soninké a été (est) illettrée et ignore les textes coraniques non rituels. Voir la conclusion de l'article. 
la médecine, les sciences de la nature, la magie-sorcellerie (sihr) et l'art talismanique (at-tilasmât)". Puis il continue:

"Si on consulte leurs ouvrages, ils croient à des propriétés (khawâss) autrement plus étonnantes que celles inhérentes à la Prophétie ( $a^{c} j a b$ min hada). Par exemple, parmi les propriétés étonnantes pratiquées dans le traitement d'un accouchement à l'expulsion difficile, on trouve cette figure (ash-shakal):

\begin{tabular}{|c|c|c|}
\hline$(4$ & 9 & 2 \\
\hline 3 & 5 & 7 \\
\hline 8 & 1 & $6)$ \\
\hline
\end{tabular}

\begin{tabular}{|c|c|c|}
\hline$d$ & $t$ & $b$ \\
\hline$j$ & $h$ & $z$ \\
\hline$h$ & $a$ & $w$ \\
\hline
\end{tabular}

Elle est transcrite sur deux morceaux de tissu sec posés sous les pieds de l'accouchée qui les fixe du regard: aussitôt, l'enfant s'empresse de sortir. Ceci est bien écrit en place et on peut le consulter dans l'ouvrage intitulé "Propriétés étonnantes" ('ajâ'ib al-khawâss)". Il s'agit d'une figure à 9 "maisons" qui contiennent des valeurs dont la propriété (ruqûm makhsûsa) est d'aboutir à un total de 15, qu'on les additionne en ligne, en colonne ou en diagonale. Ah! comment quelqu'un peut-il croire à cela et n'avoir pas l'esprit assez large pour admettre que l'appréciation de la prière légale du matin, qui est de deux prosternations, de celle de midi, qui est de quatre et de celle du soir, qui est de trois, comporte des propriétés cachées et une cause - les différents moments de la journée - non accessibles à la raison mais perceptibles seulement à travers la lumière de la Prophétie!" (Munqid, 66-67)

Petite clarification. La "figure" représentée - il n'y en a qu'une en réalité - est un carré constitué par les premières lettres de l'ancien alphabet arabe à usage numérique, dit abjad (cf. Colin, 1975). Si on lit les lettres suivant l'ordre de leur valeur numérique paire ( $c f$. le carré de chiffres voisin), on obtient $b d w h$, généralement vocalisé en budâh, mot qui n'a aucun sens mais que la pratique magique ultérieure a quasiment sacralisé, comme on le verra plus loin (cf. Mac Donald, 1981 et Doutté - qui vocalise badouh - 1984: 193).

L'exemple, tiré d'un ouvrage sur l'art des talismans, suggère qu'à l'époque considérée - fin XIè, début XIIème siècle - les pratiques talismaniques étaient suffisamment courantes pour qu'on s'en serve à titre d'illustration. L'objectif poursuivi, l'accélération d'un accouchement, 
paraît également correspondre, comme tout ce qui concerne la fécondation, la grossesse, la descendance, à une des demandes socialement les mieux ancrées. Notre corpus soninké, par exemple, le confirme largement.

Notons, dès maintenant, la distinction de vocabulaire établie par Ghazâlî entre sihr (magie-sorcellerie) et tilasmât (talismans, du grec d'époque hellénistique télesma $=$ rite religieux). Dans son Ihya 'c ulam ad-din, il les classe l'un et l'autre parmi les sciences "condamnables": wa ammâ al-madmûm minhu fa- 'ilm as-sihr wa at-tilasmât (Ihyâ', I, 3). On verra qu'Ibn Khaldûn a repris ces deux termes et a tenté d'étayer conceptuellement leurs différences, sans y parvenir réellement.

L'association, à partir de ce texte, du nom prestigieux d'alGhazâlî et du carré magique particulier utilisant les neuf premières lettres de l'abjad, a contribué à répandre l'image paradoxale d'un Ghazâlî magicien. L'opinion publique en est venue à estimer que l'imâm avait donné sa caution à cette recette magique, à ce carré à 3 x 3 cases qui n'est d'ailleurs plus désigné aujourd'hui que comme "mutallaț al-Ghazâlî", le carré triple d'al-Ghazâlî ! On peut même lire dans la savante Encyclopédie de l'islam (Mac Donald, 1981: 153): "après qu'al-Ghazâlî l'eut adoptée (cette formule) et désignée dans al-munqid $[\ldots]$ comme une aide inexplicable mais sûre pour résoudre les questions les plus difficiles, elle acquit peu à peu une réputation générale $[\ldots]$ et finit par devenir l'élément fondamental et l'aboutissement de toute la science des lettres ("ilm al-huraf)." E. Doutté, de son côté, constate: "El Ghazâli est une des autorités dont se prévalent constamment les auteurs musulmans de magie" (Doutté, 1981: 139, note 5).

En fait, le texte et le contexte du Munqie à propos du carré magique budah paraissent ambigus. A supposer qu'al-Ghazâlî ait eu une attitude personnelle d'incrédulité, il ne l'exprime pas ouvertement. Tout dépend en fait de l'interprétation de l'adjectif comparatif $a^{c} j a b$ : (ils croient à des propriétés) "plus étonnantes" (que celles liées à la Prophétie). Est-ce le simple constat d'une hiérarchie dans l'étonnement ou une allusion au caractère plutôt invraisemblable de ces propriétés? D'un autre côté, le raisonnement analogique utilisé: "vous croyez à cela, alors pourquoi pas à ceci?" n'est pas très convaincant si le 
premier terme, c'est-à-dire l'efficacité du carré magique, n'est pas jugée crédible. Enfin, il est probable que la simple présence d'une recette et d'un carré magiques dans un ouvrage d'al-Ghazâlî ait suffí pour les couvrir, auprès du plus grand nombre, de l'autorité de l'auteur qui a même pu être considéré comme leur auteur ${ }^{4}$ !

Plus fondamentalement, une analyse du texte original du Munqid, dans son ensemble comme dans les passages incriminés, fait apparaître le rôle sémantique crucial des termes khawâss, makhsûs, khâssa, khassîya, etc. qui donnent un surplus de sens, généralement causal, à des actes, à des objets, à des textes, à des personnes. Le carré magique possède ainsi "des propriétés étonnantes" (khawâss cajîba). L'œil de la Prophétie a des perceptions "spéciales" (khâssa). Il y a les "vertus" (khawâss) des médicaments mais aussi des étoiles. Les prescriptions de la $\operatorname{sharî}^{c} a$ possèdent de la même façon des "vertus" pour la médecine des cours. Les chiffres du carré sont "dotés de propriétés ou de pouvoirs particuliers" (makhsâs). Il faut la lumière prophétique pour percevoir les propriétés spéciales (khawâss) du nombre des inclinaisons durant la prière légale. Enfin, les visions du dormeur peuvent donner une toute petite idée des pouvoirs particuliers (khassîya) du Prophète.

Si on suit al-Ghazâlî, toutes ces propriétés témoignent d'un certain niveau d'efficacité qui se situe "en dehors de la raison ou de l'intellect (al- ${ }^{c} a q l m a^{c} z \hat{a l}{ }^{c}$ anhâ)". Cette formule symptomatique est utilisée à plusieurs reprises. Or c'est justement dans cette brèche des "propriétés merveilleuses qui sont inaccessibles à la raison" que vont s'engouffrer des magiciens musulmans comme al-Bûnîs. La dette de ce dernier

${ }^{4}$ Contrairement aux éditions arabes, la traduction française de l'édition de Beyrouth omet de reproduire graphiquement le carré magique en question.

5 La notion de khâssa (pl. khawâss) est un héritage grec (idiôma) dont le sens, à l'époque hellénistique, est passé de "propriété" d'un élément à "propriété occulte" ou "qualité sympathique" des éléments, désignant ainsi des pouvoirs réels mais cachés et inexplicables. Al-Ghazâlî n'est certes pas le premier penseur musulman à reprendre cette notion. Toute une littérature de science naturelle, alchimique ou mystique l'a précédé sur ce sujet. Mais cette conception a été centrale dans son combat contre la pensée philosophique rationaliste et lui a servi d'assise démonstrative dans la mise en place d'un système religieux mystique. Idéologiquement et sociologiquement, le succès de l'idée lui revient: Ibn ${ }^{\mathrm{c}}$ Arabî et al-Bûnî sont deux de ses disciples notables. Voir Ullmann M. (1978, IV: 1128). 
vis-à-vis des découvreurs et dévoileurs de mystères (al-ghayb) et d'al-Ghazâlî en particulier est immense.

Les titres des 40 chapitres du Shams al-ma ârif d'al-Bûnî, qui font écho aux 40 "livres" de l'lhyâ' d'al-Ghazâlî, soulignent l'importance de deux notions voisines: al-khawâss, c'est-à-dire les propriêtés occultes de tel ou tel élément, objet, entité, lettre, chiffre, mot, combinaison et d'autre part al-asrâr, c'est-à-dire les secrets ou les pouvoirs secrets des mêmes éléments. Citons seulement quelques têtes de chapitre du Shams: "Les secrets (asrâr) de la basmalat, ce qu'elle contient de pouvoirs particuliers (khawâss) et de baraka cachée"(Ch. 5). "Les vertus spéciales (khawâss) des incipit du Coran et des versets révélateurs"(Ch. 9). "Les secrets de la fâtiha, ses $d u^{c} \hat{a}^{\prime}$, ses pouvoirs particuliers célèbres (khawâssuhâ al-mashârât)"(Ch. 10). "Les pouvoirs particuliers (khawâss) de certains carrés magiques et talismans (tilasmât) bienfaisants"(Ch. 19).

Al-Bûnî prolonge, exploite et subvertit tout à la fois le domaine d'outre-raison ouvert par al-Ghazâlî à partir de cette notion charnière de al-khawâss.

On pourrait en dire autant de l'usage d'al-asrâr (sg. sirr) valorisé par al-Ghazâlî à propos des "vertus particulières ou cachées" du rituel islamique légal. Il suffit de lire les intitulés des "livres" correspondant de l'Ihyâ': kitâb asrâr at-tahâra (les secrets de l'ablution-purification); kitâb asrâr as-salât (les secrets de l'office de la prière); kitâb asrâr az-zakât (les secrets de l'impôt-aumône), etc.

Nous ne sommes dès lors pas étonné de retrouver al-Ghazâlî cité dans le cortège des maîtres enseignants (sanad mashâikhinâ) par lequel al-Bûnî légitime de façon traditionnelle son savoir: dans une des chaînes de ses maîtres en science des lettres, Muhammad al-Ghazâlî apparaît au 10ème rang (chronologique?) suivant Abû Najîb as-Suhrawardî (m. 1168) et précédant, entre autres, al-Junayd al-Bagdâdî (m. 911), Sarî ad-dîn as-Saqatî (m. 867) pour aboutir, à la fín, à Abû l-Hasan al-Basrî (m. 728) (Shams, IV, 530). Chaîne de transmission mystique, à la vérité, et non de sciences occultes.

Promoteur de la revivification du religieux islamique, notamment par l'importance accordée à ses "mystères" (asrâr et khawâss), 
al-Ghazâlî a donc été aussi le promoteur, probablement malgré lui, du carré magique budah (celui du Munqią) et à travers ce dernier, du carré magique en général.

Al Bûnî n'a pas été en reste pour honorer son maître ès sciences des lettres ${ }^{6}$ et il lui consacre dans la quatrième et dernière partie du Shams quelques budâh à sa façon. En voici un, de 16 cases, uniquement constitué des quatre lettres en question?. Al Bûnî le préconise dans le cadre de l'utilisation des bienfaits de la lettre "b" (début de budâh) et il doit permettre "d'obtenir le consentement total de la totalité de la création"! (Shams, IV, 401). Un carré identique est proposé tout à la fin de l'ouvrage (Shams, IV, 526), pour un usage au choix de l'utilisateur d'abord et à des fins amoureuses ensuite. Nous traduisons ces dernières prescriptions car il nous semble qu'elles permettent de saisir certains mécanismes du système magique d'al-Bûnî. En même temps, on y trouve une sorte de jeu de mot, volontaire ou involontaire, qui ressemble fort à un clin d'œil adressé au maître al-Ghazâlî...

"Paragraphe des carrés magiques (makhsâsa) à effets bienfaisants divers.

Parmi ceux-ci, se trouve le carré construit avec les quatre lettres b, d, w et $h$. Il faut le transcrire en deux exemplaires sur une peau de gazelle (ghazâl), un vendredi au moment du lever du soleil puis le parfumer avec du baume (lubân), de l'ambre ('anbar) et de l'encens (madd) et enrouler les deux figures (le carré budâh est reproduit deux fois dans le texte) sur une baguette de grenadier avec un morceau de soie blanche, après avoir écrit le nom du demandeur et de l'objet de la demande.

S'il s'agit d'aboutir à un mariage ou à des fiançailles (khitba), il faut prendre une colombe blanche, écrire le carré budah, c'est-à-dire la formule magique (al- $\left.{ }^{c} a z i m a\right)$ comme c'est indiqué, l'attacher à l'aile de la colombe puis envoyer celle-ci avec un

${ }^{6}$ Le Shams est une réplique formelle de l'Ihyâ en ce que, comme cette dernière, il est constitué de quatre parties et de 40 chapitres, qu'il reprend la terminologie en asrâr et khawâss, qu'il fait allusion aux paroles de 'Isâ (Jésus) pour ressusciter les morts, qu'il use de différents carrés magiques à base de budûh, etc.

${ }^{7}$ La disposition des quatre lettres $b, d, w, h$, à l'intérieur du carré, est différente dans les éditions du Munqid et dans celles du Shams. Pour arriver à lire $b(u) d u \hat{h}$ dans le Munqid, il faut lire en ligne alors que dans le Shams il faut lire en colonne. 
messager: le messager s'arrêtera devant la porte, alertera les gens de la maison puis lâchera la colombe et chaque fois que celle-ci passera en vol, la femme sera troublée (hâjat al-mar'a). Si la colombe est lâchée à l'intérieur de la maison fermée, c'est encore mieux et plus efficace."

On découvre dans la dernière recette un procédé de magie sympathique basé sur l'analogie entre les battements d'aile de la colombe et l'émoi de la femme; le symbolisme de la virginité féminine représentée par "une blanche colombe" est en partie transculturel.

Dans la premiere prescription par contre, on voit surtout apparaître un jeu de concordances entre des lettres, des moments, des parfums, des objets, tout à fait représentatif de la manière de faire d'al-Bûnî; on aura remarqué au passage que la peau sur laquelle il faut écrire le carré budûh correspond au nom de Ghazâli, à la dernière lettre près...

Un dernier mot sur budah qui continue à marquer de son empreinte nombre de talismans contemporains et qui s'adapte aux situations politico-culturelles nouvelles comme en témoigne cet exemple africain, d'époque coloniale, où budah est transcrit en valeurs numériques, tantôt suivant la graphie arabe habituelle tantôt suivant la graphie européenne ${ }^{8}$.

\begin{tabular}{|c|c|c|}
\hline$\Sigma$ & 9 & 2 \\
\hline 3 & 0 & $V$ \\
\hline$\Lambda$ & $I$ & 7 \\
\hline
\end{tabular}

\begin{tabular}{|l|l|l|}
\hline$\Sigma$ & 9 & 2 \\
\hline 3 & 5 & 7 \\
\hline 8 & 1 & 6 \\
\hline
\end{tabular}

${ }^{8}$ Talisman du Fonds Joire, Centre de Recherches Africaines, aimablement communiqué par Jean Boulègue. Apparemment destiné à la protection contre des menées magiques, le talisman comprend, outre le double tableau chiffré de budûh, 9 noms sans signification apparente (noms de jinn ou assimilés?) et surtout les deux sourates coraniques dites protectrices (CXIII et CXIV) dont l'une fait explicitement allusion à "celles qui soufflent sur les næuds", c'est-à-dire aux magiciennes ou jeteuses de sorts. 
Après l'examen de ces quelques exemples, essayons de caractériser les procédés d'al-Bûnî.

Son système peut fondamentalement être décrit comme un ensemble de rapports, sans causalité précise, entre divers niveaux et séries d'éléments qui définissent une structure générale de l'univers, faite de correspondances, sans hiérarchie ni préminence notoires. Les éléments eux-mêmes sont constitués de séries rangées ou ordonnées. Citons-en quelques-unes, en guise d'inventaire non exhaustif: les lettres de l'alphabet, le système numérique, le monde d'en-haut ('alawî) et d'en-bas (suflî), les sept planètes, les signes du zodiaque, les quatre éléments, les rites et le calendrier musulmans, le Coran, les noms d'Allah, les parfums, les couleurs, les métaux, les djinns, les anges, les sept signes "cabalistiques", les saisons, etc. Ce sont les combinaisons entre les séries et leur mise en relation bi-univoque qui constituent la grammaire de cet exercice cosmologique'. L'efficacité opératoire provient directement de la rencontre des éléments à l'intérieur de la formule précise d'une "recette".

La palette d'al-Bûnî s'étend ainsi à tous les constituants du cosmos gréco-hellénistique dans lequel il injecte en supplément le système islamique. C'est sans doute au tout début du Shams, après l'introduction, que l'on perçoit le mieux sa démarche générale, lorsqu'il présente

${ }^{9}$ Si les séries d'éléments de l'univers d'al-Bûnî sont régies par des associations, ces dernières ne prennent cependant jamais l'allure de véritables combinatoires, au sens mathématique du terme. Il n'y a pas de croisement systématique de tous les éléments d'une série avec tous les éléments d'une autre. Au contraire les éléments ont entre eux des liaisons univoques et stables; par exemple telle lettre de l'alphabet est liée à une planète bien précise ou à l'un des quatre éléments fondamentaux, de manière exclusive. Voir sur ce sujet les réflexions de D. Urvoy, 1992: 25-41. L'auteur prend des exemples chez al-Bûnî et parle fort justement, à propos des carrés magiques, de "concordances entre éléments qui, une fois transcrites sur un talisman, s'avèrent ipso facto opératoires"; il nous semble que cette affirmation peut être généralisée à l'ensemble des prescriptions d'al-Bûnî, si on tient compte du fait que beaucoup d'éléments ne sont pas transcrits mais intégrés dans le déroulement du rituel talismanique (lieu, temps, supports, etc). 
l'articulation entre cosmos, alphabet, nombres et carrés magiques (Shams, I, 5):

"Sache que les nombres ont leurs secrets (asrâr) et possedent une influence (atâr) tout comme les lettres. Le monde supérieur est la prolongation du monde inférieur. Le monde de l'arche prolonge celui du trône, lequel prolonge la planète Saturne, laquelle prolonge la planète Jupiter, laquelle prolonge la planète Mars, laquelle prolonge la planète Soleil, laquelle prolonge la planète Vénus, laquelle prolonge la planète Mercure, laquelle prolonge la planète Lune, laquelle prolonge la planete chaleur, laquelle prolonge la planète humidité, laquelle prolonge la planète du froid, laquelle prolonge la planète du sec, laquelle prolonge la planète de l'air, laquelle prolonge la planète de l'eau, laquelle prolonge la planète de la terre, laquelle prolonge la planete Saturne. A Saturne est associée dans le monde supérieur la lettre jîm, à laquelle correspond le chiffre trois ou, si on la décompose (at-tafsîl), le nombre cinquante-trois [...]; à Saturne est associé le carré magique $3 \times 3$ (al-mutallat), le plus connu parmi les experts ${ }^{10} . "$

On pourrait résumer très simplement ce texte par la formule triviale: tout se tient ! Mais on pourrait également faire appel au principe de contiguité qui semble pouvoir (ou vouloir?) expliquer le sens et l'efficacité des relations. Ce principe joue en magie, comme dans toute logique symbolique, un rôle considérable.

Fait remarquable, la seule note islamique explicite de ce texte est fournie par "les mondes de l'arche et du trône" (Cor. XXI, 22; IX, 129; II, 255 etc.) situés par al-Bûnî au-delà du monde planétaire. Quant à l'alphabet arabe, fait-il référence simplement à lui-même ou

${ }^{10}$ La clarté des enchaînements entre les mondes et les planètes, celle des associations de planètes, de lettres et de nombres n'est pas totale. On a d'un côté la grande division entre les mondes supérieur et inférieur puis, de l'autre, la cascade des planètes accrochées aux mondes du trône (kursî) et de l'arche ( $\left.{ }^{c} a r s h\right)$. Nous ne savons pas quel rapport al-Bûnî établit au juste entre ces deux séries. Nous ne comprenons pas non plus très bien comment la planète Saturne qui prolonge, au départ, le monde du trône, prolonge, à la fin, la planète de la terre (at-turâb): est-ce simplement une façon de pouvoir s'arrêter en décrivant une boucle? Les rapports, enfin, apparemment différents, entre planètes, lettres, chiffres et monde supérieur ou inférieur ne sont pas non plus très évidents. Pour des suppléments d'analyse, voir Lory, 1989. Détail d'édition? la planète du feu manque dans l'énumération et aussi, plus curieusement, la planète Terre $(a l$-ard $)$... 
à la langue divine du Coran? En fait, la toile de fond, on s'en aperçoit une nouvelle fois, releve de la cosmographie grecque et l'on ne peut qu'être intéressé en écoutant A. Bernand (1991: 82) parler de la Grèce: "Dans les textes magiques grecs apparaît la croyance que le monde forme un tout divin, dont les parties sont reliées les unes aux autres par une sorte de sympathie". Pas un mot à changer dans cette proposition si on remplace "grecs" par "arabes"..

L'originalité d'al-Bûnî, au tournant des XIIe et XIIIe siècles, n'a cependant pas été de "réchauffer" les restes de la science hellénistique mais bien d'y incorporer systématiquement tout ce qui, dans l'islam, pouvait être structuré sur le modèle des séries d'éléments déjà existants.

Voici un premier exemple où s'entrechoquent données grecques et islamiques.

"Celui qui dresse un carré de $4 \times 4$ et qui y inscrit des rapports numériques le lundi, alors que la lune est en conjonction avec la planète Jupiter, à 3 degrés dans le Taureau et ce à l'heure de la lune, celui qui l'écrit après avoir fait des ablutions puis une prière de deux rak $^{c} a$ (sous-séquences rituelles), puis la récitation de la fâtiha (sourate d'ouverture du Coran), puis cent fois le verset du trône (Cor, II, 255) puis encore la fâtiha et ensuite la sourate al-ikhlâs (Cor. CXII), s'il l'écrit (le carré) sur une peau non souillée (tâhir) et s'il le porte en amulette (hamalahu), alors Allah augmentera sa compréhension, sa mémoire, sa sagesse et embellira sa destinée parmi les gens, dans le monde supérieur ( $\left.{ }^{c} a l a w i\right)$ et inférieur (suflî). S'il le donne à porter en amulette à un prisonnier, celui-ci s'échappera rapidement. S'il le suspend au drapeau de l'armée, il la fera vaincre les ennemis mécréants et ceux qui leur font allégeance. Celui qui le porte en amulette vaincra son adversaire, par la volonté d'Allah Très Haut." (Shams, IV, 526)

En-dehors de la peau qui sert de support d'écriture et du carré magique à porter en amulette, tout un rituel islamique est venu s'ajouter au système cosmologique précédent. Ce rituel n'est autre, dans le cas présent, qu'une réplique de la prière légale musulmane (as-salât), accompagnée de récitations surérogatoires de la fâtiha et surtout du verset du trône qui est peut-être le texte coranique le plus sollicité par les rédacteurs de talismans. Dans le même sens, on peut considérer la peau "non souillée" sur laquelle on écrit le carré magique comme un 
substitut du tapis ou du lieu de prière dont la pureté (tahâra) est d'obligation canonique. Quant au carré magique à inscrire sur le drapeau de l'armée "qui vaincra les ennemis mécréants", il est difficile de ne pas y voir une audacieuse inversion des faits de la célebre bataille de Qâdisîya contre les Perses (636) où un carré magique ornait le drapeau de l'armée perse "mécréante", écrasée par les soldats de l'islam (Ibn Khaldûn, 'Ibar, I, 502 ).

On en arrive ainsi aux carrés magiques "islamiques" dont al-Bûnî s'est fait le spécialiste et qui lui ont valu la célébrité. Il ne s'agit plus des carrés magiques numériques ordinaires. Ceux qu'il a développés systématiquement et qui constituent son "fonds de commerce" le plus sûr sont basés sur "les plus beaux noms d'Allah". L'exergue du Shams et de chacune de ses quatre parties résume (et légitime) le contenu de l'ouvrage par la citation coranique (VII, 180): "Les plus beaux noms appartiennent à Allah: utilisez-les pour l'invoquer." Al-Bûnî, comme tant d'autres, a suivi cette injonction à la lettre, si on peut dire, mais à sa façon qui est celle d'un virtuose. La quasi-intégralité du Shams tourne autour des noms d'Allah ${ }^{11}$, utilisés selon des procédés variés. Le plus élaboré consiste à construire des carrés magiques à partir de la valeur numérique de leurs lettres. Prenons un exemple parmi la profusion de cas présentés (Shams, II, 166).

"Paragraphe 25 des noms du Très Haut: ghafâr (qui pardonne)

Celui qui inscrit un carré (murabbaca) avec ce nom, la dernière nuit du mois, sur une feuille de plomb (rasâs) et le porte en amulette, après avoir récité (tilâwat) le nom et son nombre (correspondant), Allah fermera les yeux sur lui, sur ses injustices, et s'il est de bonne foi, dérobera aux yeux des gens les bénéfices qu'il aura pu faire dans des guerres ou dans des choses semblables. Si quelqu'un veut rétablir la vérité, on n'acceptera pas son témoignage. Et si quelqu'un veut dévoiler la vérité sur les circonstances de son origine ("de sa naissance"), ses secrets resteront cachés et il pourra se réfugier auprès d'Allah grâce à l'évocation de ce nom dont le correspondant numérique est 1281. Quant aux (autres) noms d'Allah qu'on peut tirer de ses lettres constitutives, il y en a

11 Voir D. Gimaret (1988), étude de base sur les noms d'Allah, mais qui ne prend pas en compte leur utilisation dans l'univers magique. 
deux glorieux: muqît (nourrisseur) et qâbid (saisisseur) dont le nombre (total) est 1453 . Voici le carré":

\begin{tabular}{|c|c|c|c|}
\hline$g h$ & $f$ & $\hat{a}$ & $r$ \\
\hline 4 & 197 & 999 & 81 \\
\hline 198 & 3 & 82 & 998 \\
\hline 79 & 1001 & 199 & 2 \\
\hline
\end{tabular}

Al-Bûnî s'arrête là dans la présentation de cette recette. Il faut, si on veut que tout soit clair ${ }^{12}$, transformer les lettres du nom d'Allah en valeurs numériques, soit $\mathrm{gh}=1000, \hat{f}=80, \hat{a}=1, r=200$ et on obtient alors un carré dont le total, en lignes, colonnes et diagonales équivaut toujours à 1281, "concordance" (wafq, pl. awfâq) qui est la définition même du carré magique. Il reste ensuite à expliquer le nombre 1453 (qui n'a rien à voir avec la prise de Constantinople!) issu des deux autres noms d'Allah, muqît et qâbid, de même valeur numérique que la somme des noms des lettres composant ghafâr, soit $\mathrm{gh}=$ ghayn $(\mathrm{gh}=1000, \mathrm{y}=10, \mathrm{n}=50), \mathrm{f}=f \hat{a} \quad(\mathrm{f}=80, \hat{\mathrm{a}}=1), \hat{\mathrm{a}}=$ alif $(\hat{\mathrm{a}}=1, \mathrm{l}=30, \mathrm{f}=80), \mathrm{r}=\mathrm{râ}(\mathrm{r}=200, \hat{\mathrm{a}}=1)$. Le total de cette "décomposition" (tafsîl) de ghafâr donne 1453, ce qui correspond exactement au total des lettres simples des deux noms muqît $(\mathrm{m}=40+\mathrm{q}=100+$ $\hat{\mathrm{i}}=10+\mathrm{t}=400=550)$ et qâbid $(\mathrm{q}=100+\hat{\mathrm{a}}=1+\mathrm{b}=2+\mathrm{d}=800$ $=903$ ). Le hasard a donc voulu qu'à partir de la manipulation des lettres et des chiffres du nom d'Allah ghafâr, on arrive à en

12 Dans le carré du Shams (11, 166), il y a quatre erreurs de nombres. Le total invariant du carré est également erroné, de même que le total des deux autres noms. Cette observation est généralisable à l'ensemble de l'ouuvre éditée d'al-Bûnî (Shams, $\left.M a n b a^{c} a\right)$ et E. Doutté (1984: 178, note 1) l'avait déjà signalée. Elle pose toute la question d'une édition critique qui n'existe pas. Les libraires-imprimeurs arabes se contentent malheureusement de mettre sur le marché des éditions successives strictement répétitives. Il existe une autre explication possible de ces "erreurs" apparemment systématiques: l'auteur dissimule et brouille volontairement ses formules, pour éviter probablement qu'elles ne tombent entre n'importe quelle main. Mais cette hypothèse ne pourra pas être vérifiée tant qu'une édition critique n'aura pas éliminé les erreurs matérielles de copie, de lecture ou d'impression. 
reconstituer deux autres. Al-Bûnî répétera ce procédé dans toute une série d'autres exemples.

En réfléchissant sur ce cas, on pourrait conclure à une islamisation du carré magique par l'usage des noms d'Allah et d'un certain vocabulaire, comme tilâwa qui désigne la récitation coranique. Mais on pourrait tout aussi bien renverser la proposition et parler de l'introduction de la magie en islam. Par l'action (contraignante?) sur l'un de ses noms, Allah se trouve en effet au centre d'un réseau de concordances de type magique. Ecoutons encore ce qu'on nous dit de la magie grecque à ce sujet: "Dans la magie grecque, il s'agit de contraindre le dieu par l'évocation de son nom, à produire tel ou tel résultat. De là ces litanies de noms divins..." (A. Bernand, 1990: 79). Au surplus, dans le cas présent, l'utilisation du plomb comme support de l'écriture du carré renvoie aux plus anciennes traditions de la magie. Ce dernier point invite, soit dit en passant, à ne pas faire du papier l'unique support envisageable de l'écriture talismanique ${ }^{13}$.

Que penser de ces procédés et de l'univers de référence qui les sous-tend? Nous n'avons évidemment pas fait le tour de toutes les pratiques d'al-Bûnî mais les exemples examinés permettent d'aller plus loin.

Pour cela, il faut entrer plus avant dans la pensée islamique qui, dès la révélation coranique, s'est heurtée à la notion de magie-sorcellerie. En effet, le Coran pose tout à la fois un problème théologique et ethnographique lorsqu'il promulgue l'interdit du sihr, terme qui désigne globalement un type de pratiques de sorcellerie ou de magie mais qui n'est pas suffisamment explicite pour permettre de caractériser telle ou telle croyance, telle ou telle pratique observées.

La question centrale à laquelle des légistes musulmans se sont évertués de répondre est de savoir si toutes les pratiques magiques relèvent du sihr ou bien si des distinguo peuvent être établis entre des pratiques innocentes et d'autres prohibees. Autrement dit, si on sait que ce qui est condamné s'appelle sihr, que met-on sous ce vocable?

${ }^{13}$ Par exemple, A. Epelboin a rapporté du Sénégal une collection de vêtements magiques à écriture que nous présentons en commun (Epelboin \& Hamès, 1993). 
Rappelons que le terme sihr, apparaît, sous différentes formes nominales et verbales, dans plus de 30 occurences coraniques et qu'il est l'objet de condamnations directes dans au moins trois d'entre elles, dont Cor, X, 81:

"... Moïse dit: ce avec quoi vous êtes venus, c'est du sihr qu'Allah rendra inefficace car, assurément, Allah n'avantagera pas le travail des agents de dégradation."

Réalité reconnue dans son existence et ses effets, le sihr sera donc combattu pour motif de nuisance et de désorganisation sociales. Le prototype de cette nuisance (et de sa condamnation) est clairement désigné dans Cor, II, 102: "... on apprend par quel moyen séparer la femme de son époux... celui qui achète cela n'aura pas de part dans l'au-delà". Exemple qui sera repris à l'envi par tous les théologiensjuristes statuant sur la nocivité du sihr.

Vers la fin du XIVe siècle, Ibn Khaldûn a tenté de débrouiller l'écheveau du sihr. Il a voulu voir en particulier quelles pratiques lui correspondent et finalement sur quoi l'interdit est basé. L'intérêt de ses analyses, en dehors de l'effort d'organisation de la pensée dans un domaine protéiforme, réside justement dans la connaissance qu'il a, à son époque, de l'œuvre d'al-Bûnî. Son témoignage est d'ailleurs une présomption supplémentaire en faveur de l'existence d'al-Bûnî et de ses recettes magiques dont certaines sont venues jusqu'à nous ${ }^{14}$. A l'intérieur de la muqaddima, dans la longue section ${ }^{15}$ intitulee "A propos

14 Les "éditeurs" d'al-Bûnî recopient en exergue de chacune des quatre parties du Shams son nom, la date de son décès (1225) et un résumé de l'ouvrage, tels qu'ils figurent dans une source extérieure et unique, l'encyclopédie bibliographique Kashf az-zunûn d'al-Hajjî Khalîfa, composée au Moyen-Orient seulement au 17e siècle. D'où toutes sortes de conjectures sur l'existence d'al-Bûnî et sur l'éventualité de sa fonction. de prête-nom pour des cuvres qui pourraient en réalité n'être que des compilations collectives. Le témoignage d'Ibn Khaldûn est un des éléments de réponse à ces interrogations. Ce pourrait être un sujet de thèse pour un arabisant, d'Afrique occidentale par exemple, d'essayer d'éclaircir les questions qui tournent autour de la biographie d'al-Bûnî et de ses auvres.

15 Ibn Khaldûn, Kitâb al ${ }^{c}$ ibar, muqaddima, toutes éditions (Bulâq, Quatremère, Wâfî) sauf les éditions libanaises courantes qui, pour une raison mystérieuse, ne reproduisent pas cette section, la seule où Ibn Khaldûn fait explicitement référence à a]-Bûnî. Nous avons mené de vaines recherches sur ce "mystère". 
de la science de la sîmîa", Ibn Khaldûn conclut certaines de ses analyses en disant: "c'est cela que fait al-Bûnî dans ses Anmât". Dans le Shifâ as-sâ' $i l^{16}$, qu'il a écrit avant les ${ }^{c} i b a r$, on retrouve les mêmes références et parfois même des citations directes d'al-Bûnî. En se basant sur ces passages, certains - en particulier de Slane - ont pensé que les Anmât était un livre perdu d'al-Bûnî. Or il suffit de lire le sommaire du Shams (I, 4) puis les sections correspondantes (III, 268-304) pour s'apercevoir qu'al-Bûnî a regroupé les fameux noms d'Allah en dix séries qu'il appelle namt, pl. anmât. Chaque namt traite des invocations ( $\left.d a^{c} w a \hat{t}\right)$ particulières aux noms de sa série. Il est donc plus que vraisemblable qu'il s'agisse là des Anmât auxquels se réfere Ibn Khaldûn.

Quelle analyse en propose-t-il?

Lui aussi a lu al-Ghazâlî et il commence par poser l'hypothèse qu'il y a une différence entre sihr et tilasmât. Mais, chemin faisant, son analyse se complique. En effet, dans le registre des pouvoirs "spéciaux" qu'il passe en revue, il ne peut omettre ni le Prophète et ses miracles $\left(m u^{c} j i z \hat{a} t\right)$ ni le saint (walî, voire sâfi) et ses prouesses (kara$m a ̂ t$ ). L'ensemble des éléments de son analyse à ce sujet peut être rassemblé dans un tableau (voir ci-dessous).

La symétrie de ce tableau n'échappera à personne. Elle suggère une vision dualiste du monde, avec le Bien du côté de la prophétie et de la sainteté et le Mal du côté de la sorcellerie et des talismans. En même temps il y a des degrés dans le Bien comme dans le Mal: la sainteté est une sorte de sous-prophétie du point de vue de l'étendue et de la force des pouvoirs; il en est de même pour l'art des talismans par rapport à la sorcellerie.

En fait, les apparences sont trompeuses. A l'intérieur de la symétrie est installée une hiérarchie. Le dualisme n'est pas complet. Allah n'a pas de vis-à-vis "mauvais" de même niveau, de sorte que les

${ }^{16}$ Le Shifâ as-sâ'il vient très judicieusement d'être traduit en français par R. Pérez, (cf. Ibn Khaldûn, 1991). Regrettons le titre qui n'est pas celui d'Ibn Khaldûn! La traduction de anmât par "modes" (p. 190) pourra paraître sibylline, s'agissant simplement de "séries", "regroupements", "classements". Nous avons utilisé l'édition de Khalifé I. A., 1959. 


\begin{tabular}{|c|c|c|c|}
\hline $\begin{array}{l}\text { Prophétie } \\
\text { nubûwwa }\end{array}$ & $\begin{array}{l}\text { Sorcelleric } \\
\text { wilâya }\end{array}$ & $\begin{array}{l}\text { Sainteté } \\
\text { sihr }\end{array}$ & $\begin{array}{c}\text { Talismans } \\
\text { tilasmât }\end{array}$ \\
\hline $\begin{array}{l}\text { Influence sur } \\
\text { les créatures } \\
\left(t a^{\prime} \underline{t} \hat{r} f \hat{i} l \text {-akwân }\right)\end{array}$ & $\begin{array}{l}\text { Action sur événe } \\
\text { ments de ce } \\
\text { monde (ta 'tîr } f i \\
\text { ahwâl al-calam) }\end{array}$ & $\begin{array}{l}\text { Action directe sur } \\
\text { les éléments (lâa } \\
\left.\text { yahtâj ilâ } m u^{c} \hat{i} n\right)\end{array}$ & $\begin{array}{l}\text { Action à l'aide } \\
\text { d'intermédiaires } \\
\text { (yasta }{ }^{c} \text { in bi-) }\end{array}$ \\
\hline $\begin{array}{l}\text { Action par grâce } \\
\text { divine (madâd } \\
\text { ilâhi) }\end{array}$ & $\begin{array}{l}\text { Action par aide } \\
\text { divine (bi-imdâd } \\
\text { al-ilâhî) }\end{array}$ & $\begin{array}{l}\text { Action psychique } \\
\text { de nature démo- } \\
\text { niaque ou céleste }\end{array}$ & $\begin{array}{l}\text { Action via les } \\
\text { forces astrales, } \\
\text { le secret des } \\
\text { nombres etc. }\end{array}$ \\
\hline $\begin{array}{l}\text { Action sociale } \\
\text { ment bénéfique: } \\
\text { bonnes causes } \\
\text { (sâhib al-khayr) }\end{array}$ & & $\begin{array}{l}\text { Action nuisible } \\
\text { socialement: } \\
\text { mauvaises causes } \\
\text { (sâhib ash-sharri) } \\
\text { Culte aux démons } \\
\text { et astres } \\
\text { (impiété: kufr) } \\
\text { Condamné par } \\
\text { loi religieuse } \\
\text { (sharía) }\end{array}$ & $\begin{array}{l}\text { Pouvoir par les } \\
\text { lettres, les mots, } \\
\text { sans recours à } \\
\text { la divinité: } \\
\text { sîmîâ = variété } \\
\text { de sihr } \\
\text { Condamné par } \\
\text { loi religieuse } \\
\left(\operatorname{sharíc}^{c} a\right)\end{array}$ \\
\hline $\begin{array}{l}M u^{c} j i z a ̂ t \text { (mira- } \\
\text { cles) plus forts } \\
\text { que sihr. }\end{array}$ & $\begin{array}{l}\text { Action possible } \\
\text { par les mots; } \\
\text { pouvoir donné } \\
\text { par Allah } \\
\text { (kalimât } \\
\text { imânîa })\end{array}$ & $\begin{array}{l}\text { Sihr moins fort } \\
\text { que } m u^{c} j i z a ̂ t\end{array}$ & $\begin{array}{l}\text { Plus faible que } \\
\text { sihr (adcaf } \\
\text { rutba) }\end{array}$ \\
\hline
\end{tabular}


actions prophétiques soutenues par lui l'emportent sur les actions de sorcellerie. Mais il n'empêche que la tendance à la séparation métaphysique du Bien et du Mal ne se manifeste jamais aussi nettement en islam qu'en présence des sciences occultes. Celles-ci sont dérangeantes parce qu'elles attestent de pouvoirs concurrents de ceux d'Allah, même affectés d'une efficacité moindre.

Ceci étant, la condamnation religieuse n'épargne pas l'art des talismans et l'atteint par le biais de la sorcellerie dont elle serait une dérivation, aux moyens différents et plus faibles mais de nature identique. Ibn Khaldûn a bien conscience de la tentative d'al-Bûnî qui, dit-il, voudrait pratiquer une magie sans hérésie, en faisant appel à des invocations, à des noms, à des paroles tirés du Coran et de la sunna, en parlant non pas de magie mais de sîmîyâ (science du secret des lettres). Hélas! tout cela n'est qu'habillage et apparences. La base du système reste l'astrologie et ses moments favorables et l'on est donc bien en face d'une variêté de sihr, c'est-à-dire de concurrence à Allah. "Si l'on feuillette, écrit-il, les Anmât [d'al-Bûnî] et les invocations qu'elles contiennent, [on constate que] leur classification repose sur la périodicité des sept planètes..." (Shifâ, 55); ou encore, plus généralement: "... on en revient aux fondements de leur voie magique (tarîqa sihrîya) qui est le rapport des astres avec la totalité des êtres du monde, essences et contingences, substances et sens, lettres et noms. Et à chaque astre est attachée spécifiquement une partie d'entre ceuxci" (Shifâ, 55). C'est pour cela, conclura-t-il, que "la sharî́a ne fait pas de distinction entre sihr et talismans et qu'elle range tout ça dans le chapitre unique des choses défendues (mahzûran)" ("Ibar, I, 502).

L'argumentation n'est d'ailleurs pas uniquement religieuse. La $\operatorname{sharl}^{c} a$, explique encore Ibn Khaldûn, condamne "la sorcellerie, les talismans, l'illusionnisme" parce que leurs effets sont nocifs au sein de la société. Le sorcier est par excellence l'homme des mauvaises causes: tuer, annihiler des gens ou des animaux, séparer des couples mariés, favoriser des voleurs, etc. Ce point de vue n'est pas particulier à Ibn Khaldûn, malgré l'attachement de ce dernier à la maslaha malikite (intêrêt général). Al-Ghazâlî l'énonce déjà clairement dans l'Ihyâ': 
"Sache que la science n'est pas condamnable en elle-même (lâ yadumm $l i-^{c}$ aynihi) mais en raison des hommes (al- $\left.{ }^{c} i b a \hat{a}\right)$, pour l'une des trois causes suivantes: 1) quand la science se fixe le préjudice (darar) comme objectif, du fait de son détenteur ou de quelqu'un d'autre; sont condamnables à ce titre la sorcellerie et les talismans, réalités bien attestées par le Coran; c'est un de leurs effets que de séparer des gens mariés, par exemple, et ce fut aussi le cas de l'ensorcellement du Prophete, qui en tomba malade..." (Ihyâ', III, 31).

On note qu'al-Ghazâlî, de façon encore plus marquée qu'Ibn Khaldûn, se réfère au Coran plutôt qu'à une réalité sociologique empirique pour démontrer la nocivité sociale de la magie-sorcellerie.

La concordance entre loi islamique et loi sociale qui ressort de ce raisonnement n'est pas une surprise. Elle est le postulat sous-jacent à toute pensée islamique d'époque classique. Selon ce postulat, les lois naturelles, y compris sociales - celles précisément qu'Ibn Khaldûn a développées - suivent les mêmes préceptes que la loi coranique. Tout ce qui est mauvais socialement l'est également religieusement et vice versa; cela ne peut pas ne pas être autrement. L'explication n'en est pas donnée ouvertement mais on subodore qu'elle tourne autour de l'existence d'un dieu unique qui pense ou crée de façon totale et coherrente.

Les positions de la pensée islamique sur les phénomènes de magie et de talismans nous obligent ainsi à nous interroger sur les pratiques contemporaines correspondantes. Surtout, face à l'accusation religieuse d'Ibn Khaldûn, que pouvons-nous dire de la pratique de l'astrologie aujourd'hui? Les marabouts ouest-africains, principaux pourvoyeurs de textes talismaniques, ont-ils encore des connaissances astrologiques et si oui, par le biais de quelles sortes d'enseignement et de tradition?

En parcourant le corpus soninké sous l'angle des coordonnées de temps, on s'aperçoit que celles-ci sont formulées en fonction du calendrier et des rites islamiques (vendredi, prières canoniques), à une exception près - le ler jour du mois - qui paraît sans rapport avec les configurations astrales. D'autres talismans ouest-africains, de provenances diverses, semblent aller dans le même sens (cf. Epelboin et Hamès, 1993). 
Avec la prudence qui convient, notamment en l'absence d'enquêtes approfondies de terrain, on formulera donc l'hypothèse que les moments désignés comme favorables par les talismans ouest-africains contemporains révèlent une islamisation des structures et des rythmes temporels et qu'en conséquence, ils ont - jusqu'à quel point? - perdu les références astrologiques du modèle d'al-Bûnî. Si cette hypothèse se vérifiait, cela voudrait dire que si la magie est à un moment donné entrée en islam, elle s'est en retour et à son tour très fortement ou même totalement islamisée. Mais la vérification de cette hypothèse demanderait la prise en compte de tout ce qui, dans la prescription d'un talisman, relève des consignes et des recommandations orales ainsi que des rituels accomplis au cours de la consultation elle-même, bref de tout ce qui est extérieur au contenu proprement dit de l'écrit talismanique. Rappelons que les recettes talismaniques, même si elles figurent dans des écrits ou des ouvrages disponibles, sont toujours prescrites par un spécialiste, membre de la classe sociale religieuse. Cette prescription n'est qu'un moment et un épisode d'un rituel beaucoup plus large. Par exemple, le prescripteur, avant de choisir et de confectionner le talisman, pourra être astreint à des actes de purification spirituelle et corporelle tels que des jeûnes, des exercices de chapelet (subha), etc. De même, le consultant, en utilisant le talisman, devra peut-être accomplir certains actes, observer certains interdits. Faute d'une connaissance suffisante de l'ensemble des éléments d'une consultation-prescription et de son évolution socio-historique, il paraîtra prudent de parler en termes d'hypothèses quant à la disparition ou raréfaction des références astrologiques dans l'art talismanique ouestafricain. Il serait intéressant dans cette perspective, de pouvoir établir des comparaisons avec d'autres procédés "magiques" comme la divination ou la géomancie.

L'autre grande question concerne la fonction sociale des talismans, à travers les objectifs poursuivis par les demandeurs. S'il est impossible de sortir de l'ambiguïté de la notion de "nuisance sociale" dans le cadre d'une société abstraite, on peut cependant retenir l'idée de réaction agressive qui, en dehors de tout jugement normatif, est présente dans une minorité de nos textes. Il s'agit toujours d'actions "contre un adversaire", provoquant des troubles divers (parole 
perturbée, cf. plus haut) ou entraînant sa mort, voire son émasculation (pour cause d'adultère). Dans ce cadre se situe la notion extrêmement importante de batâtâ (ou batûtâ) qui, dans de vastes régions de l'Afrique islamisée, renvoie à la magie noire ou maléfique mais aussi à une magie africaine non islamique, voire à la sorcellerie.

La majorité des requêtes talismaniques concerne l'amélioration ou le retournement de situations défavorables (amour, santé, naissance, richesse, statut social, capacités intellectuelles, etc) et l'évitement de malheurs aléatoires (maladies, tyrannies, agressions, famine, etc. ${ }^{17}$.

Les réponses et les hypothèses émises à propos de ces deux questions amènent à se tourner vers une source d'inspiration islamique qui a pu influencer l'écriture talismanique, concurremment à celle d'al-Bûnî. Il ne faut d'ailleurs pas caricaturer ce dernier; certaines de ses invocations ( $d a^{c}$ wât, adkar) trouveraient sans peine leur place dans un livre de piété ordinaire, tel ce petit exemple qui débute la dernière section de l'ouvrage (Shams, IV, 510): "Commençons par les noms du Très Haut, le savant, le sage ; celui qui les répète sans cesse, Allah satisfera son désir (mâ yurîdu), lui donnera la compréhension de la sagesse (al-hikma) et de la création divine (as-sinấat al-ilahîya)".

En fait, comme nous l'avons vu, al-Bûnî prenait lui-même modèle sur al-Ghazâlî et il s'est servi des notions de $d u^{c} \hat{a}^{\prime}$ (pl. $d a^{c}$ wât ou $a d \hat{l} \hat{l} \hat{a}$ ) et de $\underline{d i k r}$ (pl. adkar) que ce dernier avait fortement remises en honneur. Il nous semble que c'est particulièrement dans les règles et le contenu de ces "rappels et appels" (adkar wa dó wât) ${ }^{18}$, rassemblés par al-Ghazâlî dans le livre IX de l'Ihyâ, qu'on peut trouver les références d'un deuxième modèle de talisman.

${ }^{17}$ On peut comparer avec l'Egypte gréco-romaine des premiers siècles (avant et après J.C.): "Quand on lit le recueil des Papyrus grecs magiques, on constate avec surprise que les charmes guérisseurs y figurent en beaucoup plus grand nombre que les charmes malfaisants" (A. Bernand, 1991: 327).

18 C'est le titre du livre IX, divisé en cinq chapitres (lhyâ', IX, 209-289). Pour l'édition utilisée, voir la bibliographie en fin d'article. 


\section{La prière de type al-Ghazâlî}

Le livre IX commence par l'exposé de ce qu'il faut appeler un rituel rogatoire. Dès les premières lignes (Ihy $\left.\hat{a}^{\prime}, \mathrm{IX}, 259\right)$, surgissent deux notions-clé: premièrement, celle de "l'utilité (fâ'ida) des prières, globalement et dans le détail" et deuxièmement, celle du sentiment de leur efficacité, fondée sur l'assurance coranique: "Faites appel à moi, je vous donnerai satisfaction" (Cor, XL, 60) et: "Vrai, je suis proche, et lorsque quelqu'un m'invoque, je réponds à sa prière" (Cor, II, 186).

Utilité, efficacité: ces qualités des prières $\left(d u^{c} \hat{a}\right)$ ne sont pas données immédiatement; elles nécessitent la mise en place d'un rituel marqué par des contraintes qu'al-Ghazâlî développe en dix points. "Ces minutieuses prescriptions, note L. Gardet (1977: 632-634), ces conditions et règles du $d u^{\hat{c}} \hat{a}$ ' visent en fait à l'entourer de garanties d'efficacité." Examinons quelques-unes de ces conditions (âdâb).

III. Condition de temps. Il y a des moments privilégiés (al-awqât ash-sharîfa) pour intervenir aupres d'Allah; il s'en présente chaque nuit (le dernier tiers de la nuit), chaque semaine (le vendredi), chaque année (le jour de ${ }^{\mathrm{c}}$ Arafat, le mois de Ramadân). Il y a aussi 15 nuits et 19 jours particulièrement favorables et "si un commerçant néglige ces périodes, il ne fait pas de bonnes affaires" (Ihyâ', X, 317).

IV. Condition de circonstance. L'environnement le plus propice est celui des prières canoniques. Ghazâlî cite une parole du Prophète: "Pendant la phase de prosternation (sujad), faites l'effort d'émettre une $d \hat{u}^{c} \hat{a}$, elle a le pouvoir (qamin) d'être exaucée" (Ihyâ', IX, 269)

V. Condition de posture: il faut "se tourner vers la qibla (direction de la $k a^{c} b a$ ) et élever les mains", ce qui correspond à la posture du début de la prière canonique.

VI. Condition psychologique: "que la demande soit catégorique (yajzim), certaine de sa réalisation et sincère dans son espérance." $(i d, 270)^{19}$. La psychologie contemporaine appliquée parlerait sans

${ }^{19}$ L'éditeur de l'Ihyâ' signale que ce hadît (parole du Prophète) et d'autres au sens voisin, ont été recueillis de la bouche d'Abû Hurayra (compagnon du Prophète) et diversement collationnés par Ibn Hayyân, at-Tirmidî et d'autres. 
doute ici de renforcement de la motivation du sujet, facteur clé de sa réussite. Mais pour apprécier tout à fait la nature de cette condition, il faut se rappeler l'attitude religieuse profonde d'al-Ghazâlî pour qui, dans la religion, la croyance est première et le savoir ou le raisonnement secondaires voire parfois néfastes.

Croire pour réussir: al-Bûnî insistera sur le même point et s'appuiera sur les mêmes paroles du Prophète, sous une forme légèrement variante ${ }^{20}$ : "[En utilisant ce livre] ta certitude doit être sincère et tu dois avoir foi dans ses vérités - le crédit accordé aux auvres vient de leur intention. Si tu as l'intention de faire un "travail", crois en lui et en la parole du Prophète: 'que personne d'entre vous ne fasse de prière $\left(\mathrm{yad}^{c} u\right)$ sans la certitude d'être exaucé.'" (Shams, I, 3).

Au-delà de ce nouveau parallélisme entre al-Bûnî et al-Ghazâlî, il faudrait interroger plus à fond la tradition islamique des sciences occultes pour découvrir l'enracinement de ce point psychologique capital de la conviction intime sur l'aboutissement de la demande. Le philosophe et astrologue al-Kindî (fin IXe siècle) écrivait dans un ouvrage sur l'influence des astres:

"Il convient également que le désir, avec les autres choses nécessaires, soit intense pour qu'il possède un effet de mouvement; car ce qu'on témoigne avec négligence ne suffit pas à l'effet du mouvement escompté. La foi dans l'effet futur est également nécessaire car celui qui désespère de l'effet sera frustré de son vœu, même s'il a exécuté avec sagesse tout le reste. En effet, la foi, c'est-à-dire un ferme espoir en l'événement désiré, constitue le nœud et l'appui du désir comme la préparation de la scamonée aide cette dernière dans son action laxative lorsqu'elle doit être donnée en remède." (Cité par S. Matton, 1977: 98)

Celui qu'Ibn Khaldûn considérait comme le dernier grand auteur en sciences occultes, l'andalou Maslama al-Majrîtî (m. 1007-8) (ou son contemporain plus ou moins homonyme Abû Maslama al-Majrîtî) ne disait pas autre chose dans le Ghayat al-hakîm (Le but du sage): "Il

20 Voir la note précédente. Le texte se trouve dans l'introduction du Shams. 
faut que l'opérateur en magie ait foi et confiance dans ses auvres et soit sans aucun doute..."21

On peut d'ailleurs penser que la persuasion et la motivation du "prieur" sortiront renforcees par la condition suivante.

VII. Condition de répétition: "Il faut se montrer insistant dans la demande et répéter (yukarrir) la $d u^{c} \hat{a}$ ' trois fois."

Pratiquement, si on prend l'exemple d'une $d u^{c} a$ contemporaine, produite et lithographiée par le milieu confrérique mouride du Sénégal ${ }^{22}$, où les formules ghazaliennes se remarquent, on apprend qu'il faut "lire cette $d u^{c} \hat{a}^{\prime}$ trois fois et (qu') Allah ouvrira les portes de l'abondance..."

VIII. Condition de la mention initiale du nom d'Allah et de son Prophète.

Autre élément clé de toute demande: ne jamais commencer par la requête elle-même mais par le nom d'Allah suivi de celui de son Prophète. Al-Ghazâlî rapporte cette tradition: "Celui qui veut qu'Allah réponde à son désir, qu'il commence par le salut sur le Prophète (as-salât calâ n-nabî) puis qu'il exprime sa demande et enfin qu'il termine par le salut sur le Prophète".

${ }^{21}$ Cité par Matton S., id. Sur l'identification de l'auteur du Ghâyat al-hakîm, voir "al-Majrîtî", Encyclopédie de l'Islam, nouvelle édition. D'après lbn Khaldûn (Shifâ, p. 55) le Shams serait une sorte de réplique du Ghâyal al-hakîm. Al-Bûnî ne revendique pas cette filiation et le nom de Maslama ou d'Abû Maslama al-Majritî ne figure dans aucune de ses généalogies spirituelles. Ce qui veut simplement dire qu'al-Bûnî a voulu légitimer son entreprise par une chaîne de garants mystiques reconnus et non par une chaîne d'auteurs de science occulte.

22 Il s'agit d'une petite brochure de 15 pages dont 3 en wolofal (langue wolof transcrite en arabe) et 12 en arabe. Elle s'intitule Hada l-kitâb sirr sûrat al-wâqica katîrat al-manafic jiddan (sic), ("Ce livre contient le secret de la sourate al-wâqica, pleine d'utilités beaucoup") s.1., s. d. Elle est signée par Adam Hanjat (?) ben a)-Marhûm ash-Shayh Ibrâhîm. Elle se donne explicitement pour une $d \mathfrak{F} a$ et le texte démarre, après les formules pieuses d'usage, par l'énumération des "mérites de cette $d u^{c} a^{\prime \prime}$ (p. 4). La sourate $a l$-wâq ${ }^{x} a$ (l'échéance) est la sourate eschatologique qui contient une des descriptions les plus circonstanciées des délices du Paradis. On trouve dans cette $d_{u^{c}}^{c} a$ la jolie expression d'un hadît cité plusieurs fois dans l'Ihyâ': "Allah lui pardonnera tout ça, même si ses péchés sont comme l'écume de la mer (wa in kânat mitla zabad al-bahr)". 
Dans la pratique, ces instructions sont scrupuleusement respectées. Les écrits talismaniques de toute nature sont ainsi généralement introduits par un bismillah ar-rahmân ar-rahîm (au nom d'Allah clément, miséricordieux) et par la salutation sur le Prophète: sallâ Allah ${ }^{c}$ alâ sîdnâ Muhammad wa calâ âlihi wa sahbihi wa sallam taslîman (salut - ou bénédiction - d'Allah sur notre seigneur Muhammad, sur sa famille, sur ses compagnons, salut le plus complet). On retrouvera cette même formule ou une de ses variantes à la fin des talismans. Le caractère stéréotypé de telles formules permet de les considérer comme des marqueurs physiques de début et de fin de document.

IX. Condition de disposition intérieure (al-adab al-bâtin). Le demandeur doit être en état de contrition, de repentir, de retour à Allah (tawba). C'est l'attitude fondamentale (al-asl) pour que la demande soit reçue, le sine qua non pour qu'elle soit exaucée. Al-Ghazâlî utilise une expression très précise: "c'est la cause immédiate de la réalisation du vœu" (huwa as-sabab al-qarîb fi l-ijaba).

On découvre ainsi dans la prière islamique de demande $\left(d u^{c} \hat{a}^{\prime}\right)$ un ensemble de contraintes qui jouent un rôle analogue à celui des prescriptions des recettes magiques et qui poursuivent le même but, celui d'une démarche efficace en vue de l'obtention d'un résultat personnel et utile. Du point de vue des bénéfices recherchés dans les $d i f \hat{a}^{\prime}$, on peut relever, à titre d'exemples, dans l'anthologie de l'Ihyâ': contrer des ennemis; apaiser la crainte qu'on a de certaines personnes; peur des orages; guérir des maladies; soulager de préoccupations diverses; accroître ses biens, ses moyens de subsistance. Mais aussi: se faire pardonner ses fautes, éviter l'enfer, aller directement au paradis (sans avoir à rendre de comptes) etc.

Voici trois $d u^{c} \hat{a}$ ' du livre IX de l'Ihyâ'. Leur similitude de construction et de contenu avec des talismans ouest-africains est frappante. - En cas de douleur.

"Si tu découvres une douleur dans ton corps ou chez quelqu'un d'autre, débarrasses t'en avec le procédé talismanique du Prophète (ruqîya rasal Allah). Lorsque quelqu'un se plaignait d'un ulcère ou 
d'une blessure, le Prophète posait son doigt par terre puis le relevait et disait: bismillah, la terre de chez nous, mélangée à la salive de l'un d'entre nous, guérit notre malade, par la volonté de notre maître." (Ihyâ', IX, 287)

Le texte arabe qui suit bismillah, un quatrain fortement rimé en - nâa, relève de l'incantation.

- La $d u^{c} \hat{a}^{\prime}$ d'Adam.

(Il s'agit d'une réponse d'Allah à Adam)."Je t'ai pardonné et personne de ta descendance ne m'invoquera à ton imitation sans que je lui pardonne, que je dissipe ses peines et ses tracas, que j'eloigne de lui la pauvreté, que je le fasse réussir en affaires comme aucun commerçant ne l'a fait et sans que je fasse venir à lui les biens de ce monde, même s'il ne le souhaite pas." (Ihyâ', IX, 279)

- La $d u^{c} a$ du pardon (al-istighfâr).

(Le Prophète a dit): "Celui qui multiplie les prières de pardon, Allah lui accordera le soulagement de toute préoccupation (hamm), le sortira de toute difficulté et lui procurera des moyens de subsistance illimités." (Ihyâ', IX, 274)

Pour clore ce chapitre des prières de type al-Ghazâlî, on pourra comparer ce qui précède avec un talisman recueilli autrefois par P. Marty au Sénégal ${ }^{23}$.

"(Amulette pour faire fortune) L'utilité est dans le nom (d'Allah) wahhâb (le généreux). Dis la prière ci-dessous sur ton chapelet puis chaque nuit récite dix mille fois "O généreux". Tu gagneras du bien sur la terre au point que les gens en seront stupéfaits, avec la grâce d'Allah. Récite ensuite encore trois fois la prière suivante "O Allah, par ton nom caché, toi le pur, le purificateur, le roi, le saint, le généreux, le vivant, l'immuable, le clément, le miséricordieux, toi qui possèdes la majesté et la générosité, exauce ma prière."

On notera simplement que ce dernier texte, a la différence des précédents, était porté en amulette dans un étui.

23 Marty P., 1914: 346 (texte français) \& 360 (texte arabe recopié). Nous avons très légèrement repris la traduction à partir de l'arabe. 


\section{Magie et prière}

La comparaison entre les recettes de type al-Bûnî et les prières de type al-Ghazâlî, ainsi que les analyses d'Ibn Khaldûn sur sorcellerie et talismans nous conduisent à des hypothèses et à des interrogations sur les notions de magie et de prière et sur leurs rapports dans la pratique des talismans en Afrique de l'ouest.

Dans un premier temps, on peut restreindre un peu plus le problème. Nous pouvons en effet suivre Ibn Khaldûn lorsqu'il définit le sorcier et la sorcellerie par leur action directe, sans recours à des instrumentations intermédiaires: c'est essentiellement "l'union d'une psyché avec une autre psyché" (ittihâd râh bi-râh) ("Ibar, I, 501 ). Autrement dit, l'esprit (ou le corps) du sorcier est l'instrument par lequel s'effectue l'acte de sorcellerie. Que l'inspiration du sorcier soit de nature particulière, démoniaque ou autre, ne change rien aux modalités de son pouvoir.

Lorsque, comme c'est le cas en Mauritanie, on accuse un esclave noir $\left({ }^{c} a b d\right)$, par sa simple volonté, de rendre malade son maître ou lorsqu'on accuse un artisan-forgeron ( $\mathrm{ma}^{\mathrm{c}} \mathrm{lem}$ ) de vampiriser (salla) quelqu'un à distance ${ }^{24}$, on est effectivement en présence d'accusations de sorcellerie.

De ce pouvoir et de ce procédé, il n'est nullement question dans l'art des talismans où, au contraire, toute l'action intentée par le demandeur est régie en-dehors de lui par des personnes et des moyens instrumentaux divers qui font appel à des puissances efficaces extérieures. La satisfaction des désirs par le recours aux talismans chemine nécessairement par des intercessions de personnes et d'entités diverses. Nous pouvons donc éliminer de notre analyse la sorcellerie proprement dite.

${ }^{24}$ Sur les pouvoirs de sorcellerie des esclaves noirs (palmeraie de Tijigja, Mauritanie) consulter: Ahmad Wuld Alamîn ash-Shinqîtî, 1958. L'accusation de vampirisme à distance (sill) a été relevée lors d'enquêtes de terrain que nous avons effectuées dans le Trârza mauritanien dans les années 1964-67. Elle fait partie de la gamme des pouvoirs occultes généralement attribués aux artisans-forgerons ( $\left.m a^{c} \hat{a} l e m i ̂ n\right)$ et, comme toute accusation de mort par sorcellerie, peut déclencher des cycles de violence basés sur les oppositions tribales. 
Reste la question de la comparaison entre les forces et les moyens mis en cuvre par l'acte talismanique et ceux des prières de demande. La structure logique et grammaticale d'une recette talismanique écrite se décompose schématiquement en trois segments: 1) celui qui veut ou désire ceci ou cela (wa man arâda an...) 2) qu'il fasse ceci ou cela $(f a-\ldots)$ 3) il obtiendra tel résultat (wa...). Cette structure est tout à fait transposable à la $d u^{c} \hat{a}^{\prime}$ et il nous semble que le schéma mental est dans les deux cas le même: accomplir scrupuleusement ce qui est indiqué pour aboutir impérativement au résultat. La mise en place du rituel rogatoire ne vise jamais qu'à obtenir de la façon la plus contraignante possible ce qui est désiré. Al-Ghazâlî, qui est un orfèvre de la réflexion sur la causalité (cf. Table ronde Unesco, 1987), ne s'y est pas trompé. La fin du livre IX sur les $d u^{c} \hat{a}^{\prime}$ tente de prévenir l'objection selon laquelle il y aurait contradiction entre l'efficacité de la prière de demande et le dogme de la prédétermination absolue de toute chose par la volonté d'Allah (al-qadâ). Autrement dit, comment peut-on provoquer l'intervention d'Allah par une $d u^{c} \hat{a}^{\prime}$, si tout est déjà décidé? On retiendra de l'argumentation théologique, l'idée que "celui qui a décrété le mal l'a décrété avec des moyens pour s'en défendre (al-ladî qadara ash-sharr qadara li-dafá ahu sababan)" (Ihyâ', IX, 289). L'effet et l'efficacité de la $d u^{c} \hat{a}^{\prime}$ sont donc bien pris en compte, ce qui nous importe ici pour définir son action et ses modalités.

Si l'on admet que la notion de contrainte vis-à-vis des forces ou des entités sollicitées représente une des caractéristiques des interventions magiques, on peut considérer que la nature des techniques rogatoires islamiques mises en ceuvre dans les $d u^{c} \hat{a}^{\prime}$ peut, suivant l'attitude du demandeur, entrer dans l'univers de la magie. Cela explique peut-être la facilité avec laquelle ces prières de demande ont pu être intégrées dans une pratique talismanique où elles voisinent avec des carrés magiques sur les noms d'Allah et l'utilisation analogique des textes coraniques.

Certes, le pouvoir d'amener Allah à décider ou à agir dans le sens d'une demande n'équivaut pas à une détermination absolue et les modalités du rituel rogatoire sont là pour le rappeler. Mais il se peut aussi, explique Ghazâlî, que la demande n'aboutisse pas, malgré toute la bonne foi du demandeur et son respect des conditions exigées. C'est 
que l'intention de la demande peut ne pas être "bonne" et donc être rejetee: "... Allah exaucera toute la demande sauf si la $d u^{c} \hat{a}^{\prime}$ contient un élément de désobéissance (illâ an yad'u fî ma asîya)" (Ihyâ', X, 318). Il y a une limitation dans la satisfaction des désirs, passant par le crible de critères religieux. On retrouve là d'une certaine façon la discrimination basée sur le résultat moral de l'action. Nous ne connaissons en effet pas de $d u^{c} \hat{a}^{\prime}$ qui ambitionne de "réduire en cendres un adversaire", de "saboter un navire en voyage", "d'incendier une maison d'injustice", de "mettre la tempête entre les gens", toutes choses que la magie "noire" d'al-Bûnî, dans ses quelques chapitres "non islamiques", sur l'usage de la cire notamment, permet (Shams, I, 86-89). En réalité, ces dernières manipulations, minoritaires chez al-Bûnî, nous renseignent plutôt sur des traditions de magie ou peutêtre même de sorcellerie qu'il a pu récupérer en-dehors de la culture musulmane et qui sont fort éloignées de ses propres élucubrations astro-islamiques.

Il faudrait encore réfléchir sur les instruments et les instances intermédiaires mis en jeu par la magie et la prière. Le rituel magique ou rogatoire peut facilement être décrit et interprété. Nous avons vu, par exemple, que la $d u^{c} \hat{a}^{\prime}$ requiert une gestuelle (mains levées), une ou des postures mais surtout et essentiellement une énonciation verbale. La magie talismanique de son côté, suppose des processus divers, essentiellement basés sur un écrit qui reste muet. La prière serait-elle orale et le talisman écrit? Ceci pose la question de la transmission matérielle et sociale des textes et des possibilités d'y accéder offertes au plus grand nombre. De ce point de vue, la situation n'a pas dû être très différente depuis les époques de l'lhyâ' (12e siècle) ou du Shams (13e siècle) jusqu'aux environs de la moitié du 20e siècle, pour ce qui concerne l'Afrique occidentale. Le savoir, consigné dans des écrits, se transmet de maître à disciple, de père en fils aussi, à l'intérieur du cercle social étroit des lettrés, c'est-à-dire de la classe religieuse musulmane. Même dans ces milieux, une hiérarchie de savoir, liée au statut social, à l'affiliation confrérique, restreint à quelques-uns la possession d'un certain nombre d'écrits, comme ce fut sans doute le cas pour l'Ihyâ' et le Shams, parmi d'autres. La transmission des textes de prière et des recettes magiques s'est donc faite sur la base d'écrits dans 
un milieu social très limité. L'accès de la masse des gens, en majorité analphabètes, à ces formules talismaniques ou à ces prières ne pouvait se faire que par l'intermédiaire de consultations auprès des spécialistes, détenteurs des textes manuscrits, recopiés ou plus rarement lithographiés. Dans le cadre de ces consultations, compte tenu toujours de l'analphabétisme du client, il ne pouvait être question ni de lui faire apprendre oralement une prière en arabe d'une certaine longueur (Coran mis à part?) ni encore moins de lui remettre le texte écrit de cette prière pour qu'il la récite au moment adéquat ${ }^{25}$. Ceci explique le caractère muet des écrits qu'on trouve dans différents types d'amulettes, même lorsqu'il s'agit de véritables $d u^{c} a$, réduites alors au sort commun des talismans à écriture.

Nous arrivons maintenant à une autre constatation. Si les $d u^{c} \hat{a}^{\prime}$ islamiques traditionnelles sont utilisées comme des techniques rogatoires magiques, à l'inverse, il nous semble que la magie talismanique de type al-Bûnî, s'est progressivement islamisée. L'univers coranique, son peuplement (anges, djinns), ses personnages mythiques (les prophètes), ses textes maniés et manipulés, les noms d'Allah, Allah lui-même, son Prophète, l'histoire islamique (les quatre premiers khalifes), le rituel islamique, son calendrier, sans oublier l'écriture elle-même et la calligraphie, tout cela constitue désormais le motif central des talismans. Il reste d'al-Bûnî l'utilisation des carrés magiques (al-awfâq), eux aussi islamisés à travers un Coran chiffré, l'utilisation de tableaux et de figures géométriques diverses, avec le reliquat de quelques signes cabalistiques dont le sens échappait déjà à al-Bûnî lui-même. Disparus apparemment les computs astrologiques et l'observation des constellations zodiacales pour déterminer les moments favorables à la confection ou à l'utilisation des talismans. Faut-il conclure aussi: disparu le gigantesque écheveau panthéiste des séries naturelles et conceptuelles, structuré par le seul jeu apparent des correspondances terme à terme?

${ }^{25}$ L'arabisation lettrée semble progresser un peu depuis 1980 , sous la pression des mouvements et associations islamistes. 
Il est probable que l'islamisation de l'art talismanique affecte la nature des demandes elles-mêmes. A l'instar des $d u \mathcal{E} \hat{a}^{\prime}$ qui ne peuvent aboutir que si elles visent un résultat conforme aux exigences religieuses, le talisman n'aurait-il pas tendance à se réduire à un champ de demandes semblables? D'où parfois un surprenant mélange de demandes concernant ad-dunyâ (la vie d'ici-bas) et al-âkhira (l'au-delà). Aller au paradis sans jugement est une requête qui est faite de la même façon et au même niveau qu'une demande d'ascension sociale, d'acquisition de richesses ou d'accroissement de la vivacité d'esprit. A travers l'islamisation des talismans, assiste-t-on à une islamisation de l'expression des besoins personnels?

Le double mouvement de "talismanisation" de la $d u^{c} \hat{a}$ ' et d'islamisation du contenu des talismans à écriture pose donc une série de questions. L'héritage astrologique d'al-Bûnî a-t-il disparu ou bien les seuls talismans sont-ils incapables de nous renseigner à ce sujet? S'il peut y avoir, à notre avis, un aspect magique dans le déroulement rituel de la $d u^{c} \hat{a}^{\prime}$, celui-ci ne s'accentue-t-il pas lorsque la $d u^{c} a$ devient talisman, c'est-à-dire texte écrit muet renfermé dans un étui, porté sur le corps, attaché à un endroit précis, lavé et bu, etc.? L'autre versant du même problème est lié à la nature des talismans à écriture. Pratique sans doute distincte de la sorcellerie, peut-on pour autant dire que l'islamisation de son contenu ait modifié l'univers mental de ses références et évacué les procédures magiques? Ne peut-on pas considérer que la démarche de type recette magique reste entière? Ces questions elles-mêmes renvoient à l'utilisation du concept de magie et à son acception. Est-ce que nous sommes capables finalement de caractériser et de délimiter conceptuellement les pratiques et les croyances dites magiques et celles dites religieuses? Peut-il y avoir, dans le milieu scientifique contemporain, un accord sur l'existence de la magie, de pratiques magiques et si oui, sur la base de quels critères? 
On voit bien que l'étude des talismans musulmans d'Afrique occidentale et de leurs modèles rend inévitable une réflexion théorique sur l'idée et les techniques de la magie, particulièrement dans le contexte islamique.

Constant Hamès

UPR 17 (Groupe de sociologie des religions) CNRS

\section{Références bibliographiques}

Bernand André

1991 Sorciers grecs, Paris, Fayard, 513 p.

Abû l- ${ }^{c}$ Abbâs Ahmad ben ${ }^{c}$ Alî al-Bûnî

s.d. Shams al-ma ârif wa latâ'if al- ${ }^{c}$ awârif (Le soleil de la connaissance et les délices des connaisseurs), Al-maktabat at-taqâfiya, Beyrût, 535 p.

Colin G.S.

1975 "Abjad", Encyclopédie de l'Islam, nouvelle édition, I, p. 100.

Doutté Edmond

1984 Magie et religion dans l'Afrique du Nord, Paris, Geuthner et Maisonneuve, 617 p. (lère éd. Alger, 1908)

Epelboin, A. \& Hamès C.

1993 "Trois vêtements talismaniques provenant du Sénégal (décharge de Dakar-Pikine)", Damas, Bulletin des Etudes Orientales.

Gardet, Louis

1977 "Du'ầ'", Encyclopédie de l'Islam, nouvelle édition, II, 632-634. 
Al-Ghazâlî Muhammad

s.d. Al-munqid $\min$ ad-dalâl, Al-maktabat al-jund s.l. (Le Caire?), édité sous le titre Erreur et délivrance, texte arabe et traduc. franç. par Farid Jabre, Beyrouth, Imprimerie Catholique, 55 et 122 p., Coll. Unesco.

s.d. Ihyâ' 'culûm ad-dîn, Beyrût, Dâr al-Qualam, 5 vol.

Gimaret, Daniel

1988 Les noms divins en Islam. Exégèse lexicographique et théologique, Paris, éd. du Cerf, 448 p.

Hamès Constant

1987 "Taktub ou la magie de l'écriture islamique. Textes soninké à usage magique", Arabica, XXXIV, 3, 305-325.

Ibn Khaldûn ${ }^{c}$ Abd ar-Rahmân

1959 Shifâ' as-sâ'il li-tahdîb al-masâ'il, introduc. et vocab. technique par Khalifé, I.A., Beyrût, Imprimerie Catholique, 189 p. (arabe) et $14 \mathrm{p}$. (français)

s.d. Kitâb al- ${ }^{c}$ ibar, muqaddima, Beyrût, Ed. al-A lamî, 7 vol.

1991 La Voie et la Loi ou le Maître et le Juriste, trad. présent. annot. René Pérez, Ed. Sindbad, 308 p.

Lory Pierre

1986 "Magie et religion dans l'œuvre d'al-Bûnî", Horizons Maghrébins (Toulouse), 7/8, 4-15.

1989 "La magie des lettres dans le Shams al-ma ${ }^{\mathrm{c} a}$ ârif d'al-Bûnî", Bulletin d'Etudes Orientales, Damas, XXXIX-XL, 97-111.

MacDonald D.B.

1981 "Budûh", Encyclopédie de l'Islam, nouvelle édition, suppl. 3-4, p. 153.

Marty Paul

1914 "Les amulettes musulmanes au Sénégal", Revue du Monde Musulman, 27.

Matton Sylvain

1977 La magie arabe traditionnelle, Paris, Retz.

Ahmad Wuld Alamîn ash-Shinqîtî

1958 Al- wasît fi tarâjim udabâ' Shinqît, 2e édition, Mu'asasat Al-Khânjî, Le Caire. 
Table Ronde Unesco

1987 Ghazâli. La raison et le miracle, Paris, Maisonneuve et Larose, $196 \mathrm{p}$.

Ullmann, $M$.

1978 "Khâssa", Encyclopédie de l'Islam, nouvelle édition, IV, p. 1128

Urvoy, D.

1992 "Sur un aspect de la combinatoire arabe et ses prolongements en Occident", Arabica, XXXIX, 1, 25-41. 